\title{
ABOUT THE INFLUENCE OF THE MACHINED DEFECT SHAPE AND POSITION ON THE STRESS DISTRIBUTION FOR PIPELINES WITH VOLUMETRIC DEFECTS
}

\author{
Ionuţ LAMBRESCU \\ Universitatea Petrol-Gaze din Ploieşti, Bd. Bucureşti 39, Ploieşti, Romania \\ e-mail: ilambrescu@upg-ploieşti.ro
}

\begin{abstract}
The aim of the paper is to analyse how the shape and position of the machined volumetric surface defects (VSD) may influence the stresses and displacements distributions for pipelines with VSD. The machined defect is usually rectangular, with edges oriented parallel/perpendicular to the pipe's axis.

We attempt to analyse in this paper what happens when the machined defect is no longer rectangular, but circular or elliptical and with different positions relative to the pipe's axis. We also consider different fillet radius of the machined VSD.
\end{abstract}

KEYWORDS: Volumetric surface defect, VSD

\section{Introduction}

In the case of pipelines with volumetric surface defects, a number of methods were developed and included in standards and normatives such as ASME B31G; ASME B31.8; API Standard 579, BS 7910.

Basically, we speak about the following approach:

- Initial anomality evaluation. The result of this stage is the conclusion that the anomality is acceptable and no further actions are necessary, or if it is considered a defect, a repairing process has to follow.

If the anomality is considered a defect, then the affected area is machined and after that, the defect area will withstand a repairing process. This process includes the use of a filler that will fill the VSD and of a wrap applied around the pipe in the VSD area [1$6]$.

In the literature for the machined VSD only a rectangular shape is considered. This is why we appreciated that attention should be given also to other shapes for the machined VSDs.

At that stage we took into consideration three shapes: circular, elliptical and rectangular. We also took into consideration, for the elliptical and rectangular machined VSDs, the orientation of the machined defect.

In order to compare the results, we considered machined defects with equal areas.
For all of the considered cases, the analysis was performed on a pipe with the following properties:

- Outside Diameter De $=508 \mathrm{~mm}$.

- Wall Thickness $\mathrm{t}=10 \mathrm{~mm}$.

- Maximum Allowable Pressure MOP $=20$ bar.

- Specify Minimum Yield Strength SMYS = $290 \mathrm{~N} / \mathrm{mm}^{2}$.

- Maximum Depth of VSD dp = 7.5 mm.

\section{Circular machined VSD}

The first machined VSD considered is a circular one, as can be seen in Figure 1. We mention that the defect is positioned at the middle of the considered pipe segment and that a fillet radius is also considered as a parameter of the evaluation. The fillet radius will vary between $7.5 \mathrm{~mm}$ (the defect depth) and $20 \mathrm{~mm}$, with a $2.5 \mathrm{~mm}$ step. Due to the circular shape of the machined VSD, there is not the case to speak about the orientation of the machined VSD. A scenario analysis produced the results presented in Fig. 2.

Figure 3 presents the Von Mises stress distribution for a quarter of the pipe (due to symmetry reasons) for the "optimal" case (with the smallest Von Mises stress). As can be seen, the maximal value is recorded on the interior face of the pipe.

The analysis of the last row in Table 1 allows us to state that the fillet radius, at least for the interval considered, does not influence significantly the stress or displacement distributions. 


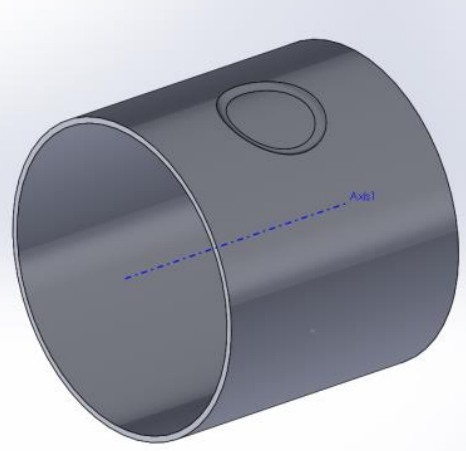

a. $3 \mathrm{D}$ view

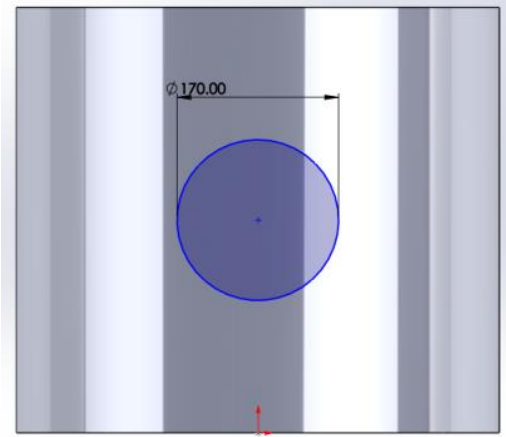

b. Top view

Fig. 1. Circular machined VSD

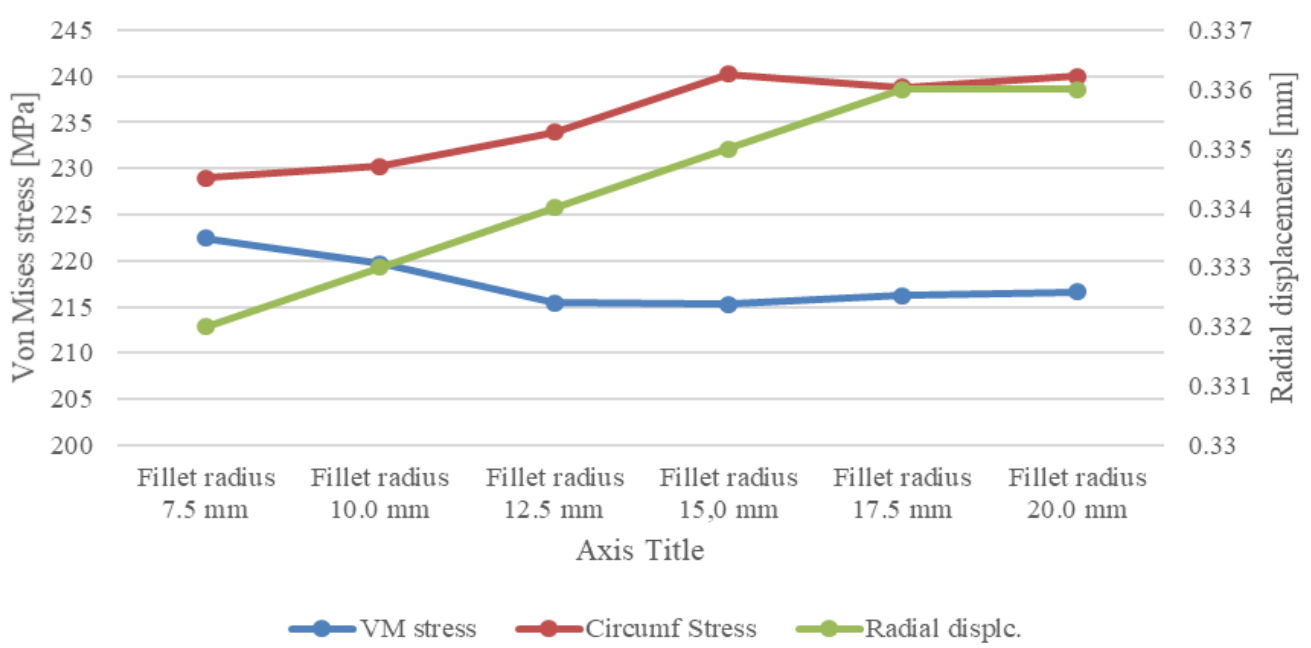

Fig. 2. Stress and displacement tendencies for the 6 scenarios

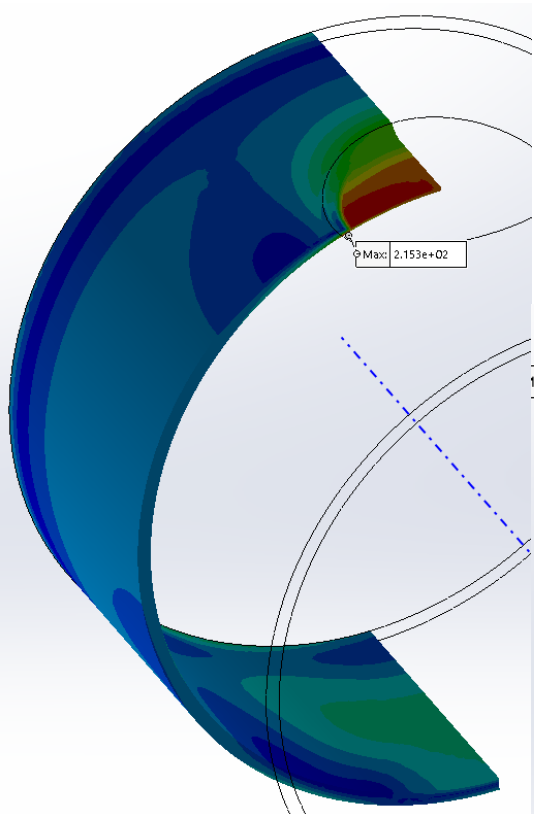

a. Section view

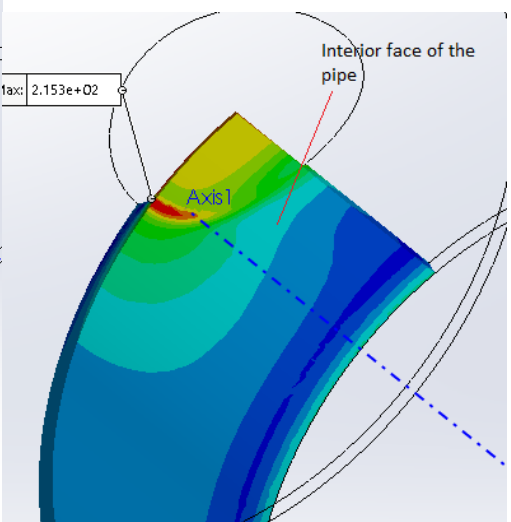

b. Detail view

Fig. 3. Stress distribution for the optimal scenario (Von Mises [MPa]) 


\section{Elliptical machined VSD}

For the case where the machined VSD has an elliptical shape, apart from the fillet radius, a new parameter has been considered. It is the position/orientation of the machined VSD. We express that using an angle, as can be seen in Figure 4. We also mention that the area of the ellipse is equal to that of the circle used for the circular machined $\mathrm{VSD}$, and that the ratio between the axis of the ellipse is 2 .

This time the scenario analysis turned into an optimization analysis, with tow parameters: the fillet radius (varying between 10 and $20 \mathrm{~mm}$ with a $2 \mathrm{~mm}$ step) and the angle that positioned the VSD (varying between 0 and 90 degrees with 10 degrees step). This approach led to 60 scenarios.
The results indicate that the minimum Von Mises stress is recorded for the ellipse positioned with the big axis perpendicular to the pipe's axis.

As for the way the fillet radius influences the results, we may say that its importance is bigger for small values of the angle. As expected, we record smaller Von Mises stress for bigger fillet radius values (see Figure 6). The most important parameter in terms of how it influences the Von Mises stress is the position angle of the machined VSD.

Figure 5 presents the Von Mises stress distribution for a quarter of the pipe (due to symmetry reasons) for the case where the Von Mises stress records the minimal value among scenarios (fillet radius $20 \mathrm{~mm}$ and orientation angle 90 degrees). As can be seen, this maximal value is on the exterior face of the pipe.

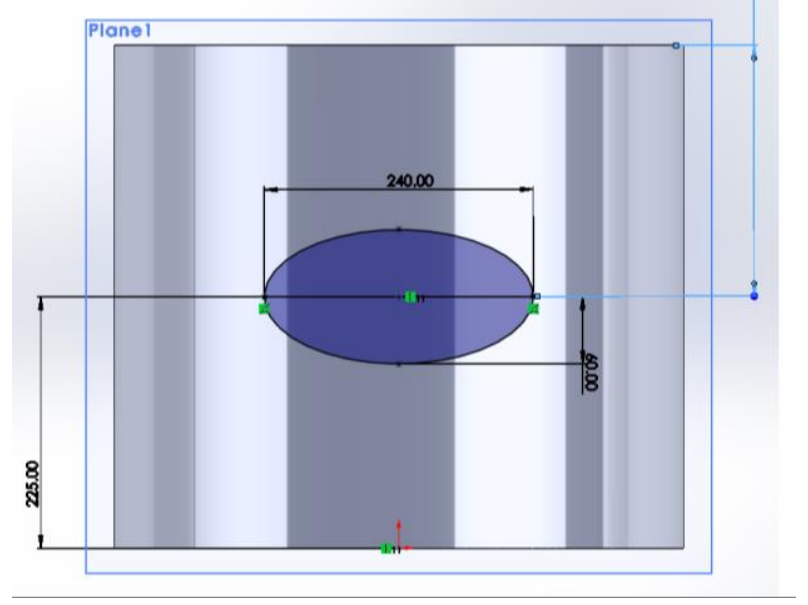

Fig. 4. Elliptical machined VSD

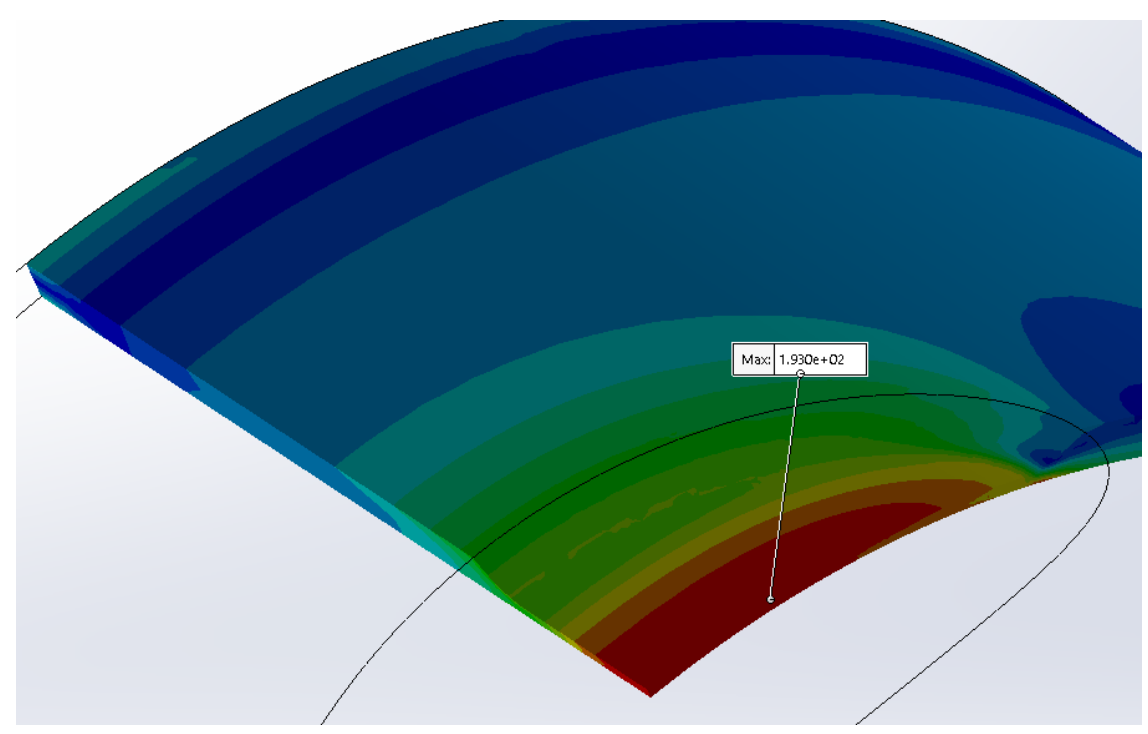

Fig. 5. Von Mises stress distribution [MPa] 

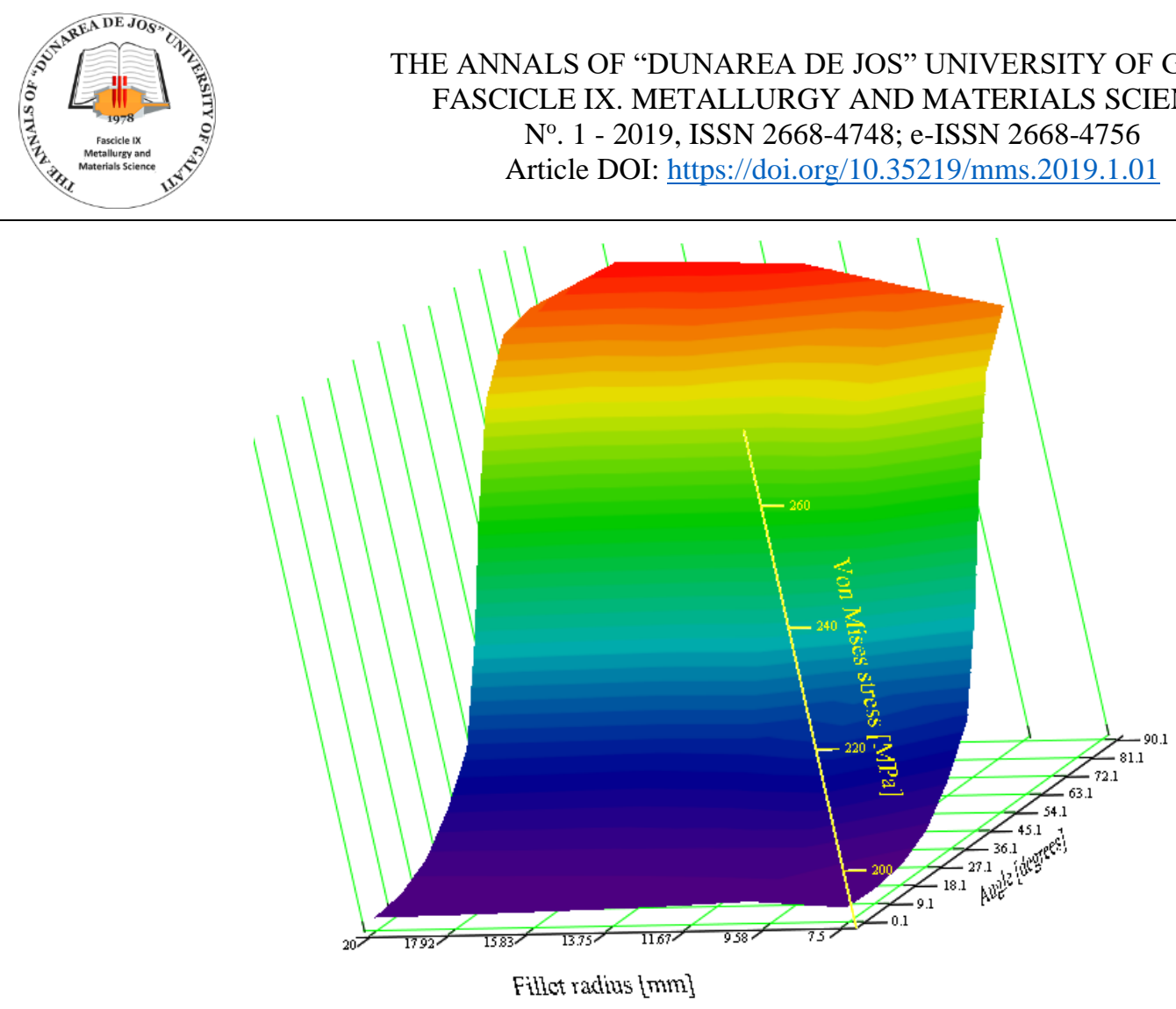

Fig. 6. Influence of Fillet radius and position angle on Von Mises stress

For the elliptical machined VSD, we can conclude that the most favourable scenario corresponds to the orientation of the major axis perpendicular to the pipe's axis and the fillet radius of $20 \mathrm{~mm}$. This position ensures the minimum length of the machined VSD along the pipe's axis.

The most detrimental situation is recorded for the VSD oriented with the major axis parallel to the pipe axis and the fillet radius of $12 \mathrm{~mm}$. We have to mention that for the case when the VSD is oriented along the pipe's axis, the value of the fillet radius has not a significant influence (relative difference of $1.39 \%)$.

In the meantime, we stress that the relative difference between the most detrimental and the most favourable scenario, for the Von Mises stress, is $40.85 \%$, which indicates a very strong influence of the VSD orientation on the stress distribution.

\section{Rectangular machined VSD}

We considered a rectangular VSD with the same area as the previous two types (circular and elliptical), with a ratio between the rectangle dimensions of 2 . We run 60 scenarios, corresponding to the variation of the fillet radius between 10 and $20 \mathrm{~mm}$, with a step of $2 \mathrm{~mm}$, and of the orientation of the VSD with an angle between 0 and 90 degrees, with a 10 degrees step (see Figure 7).

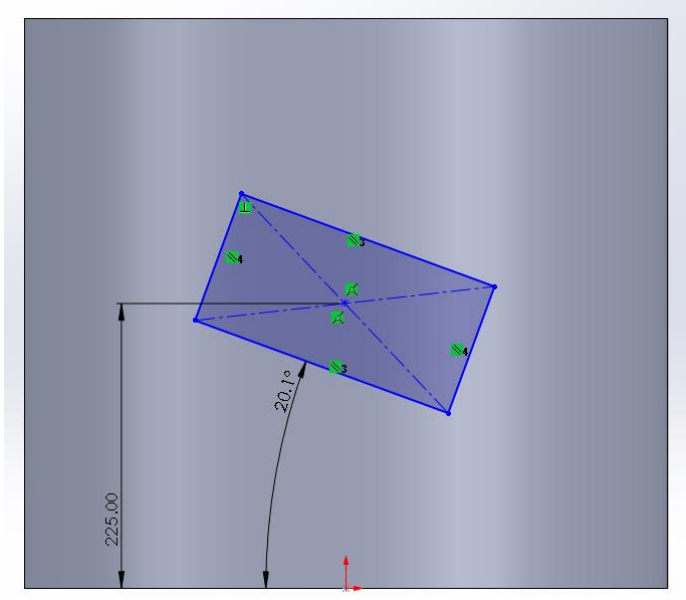

Fig. 7. Machined VSD position 
The first round of the 60 scenarios produced a most favourable situation (the smallest Von Mises stress) for the case when the fillet radius is $14 \mathrm{~mm}$ and the orientation of the VSD is 20 degrees. The most detrimental situation is recorded when the VSD is aligned with the pipe's axis and the fillet radius is $16 \mathrm{~mm}$.

The relative difference between those two extreme scenarios is $47.13 \%$.
Figure 8 presents the Von Mises stress distribution for the most favourable scenario in a section along the VSD axis, while Figure 9 presents the Von Mises stress distribution for the most detrimental situation.

Figure 10 presents the variation of the Von Mises stress for the 60 scenarios. One can see a region with smaller values for the Von Mises stress around the value of 20 degrees for the VSD position.

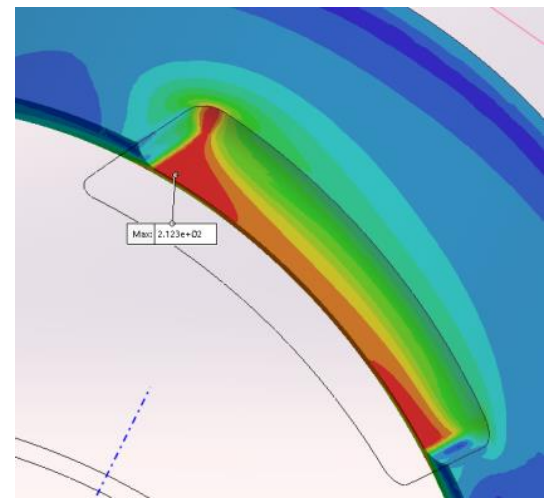

Fig. 8. Von Mises stress distribution for the most favourable scenario [MPa]

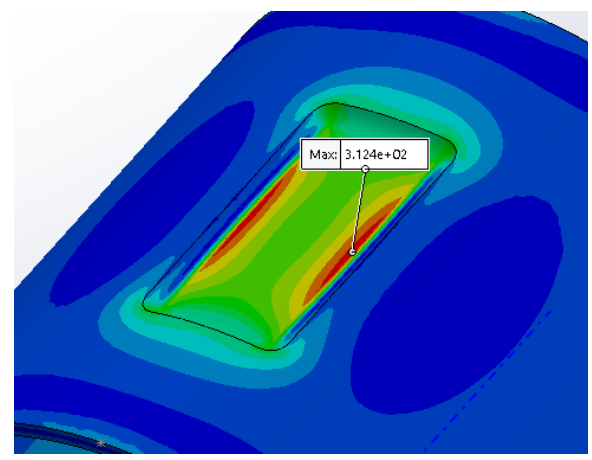

Fig. 9. Von Mises stress distribution for the most detrimental scenario [MPa]

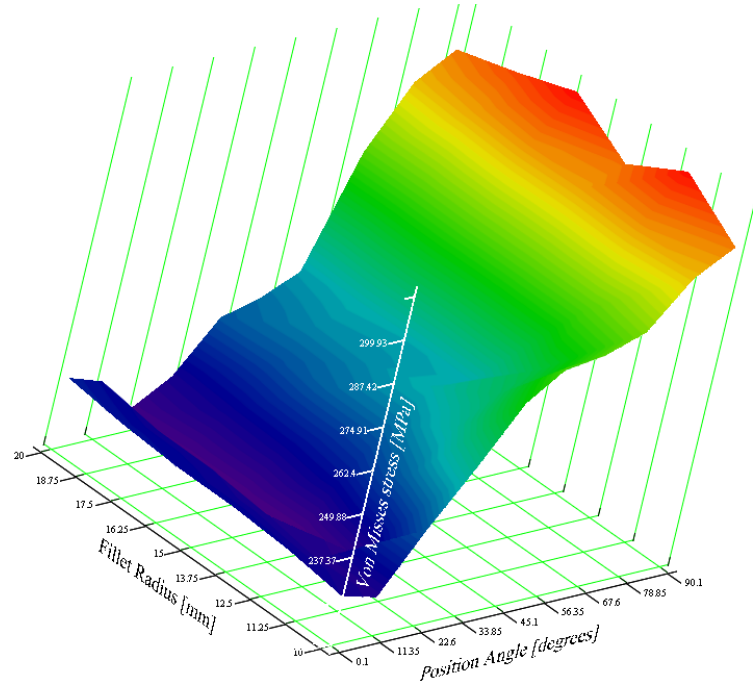

Fig. 10. Von Mises stress distribution for the 60 scenarios 
For the rectangular VSD, we also considered other scenarios, that would analyse the cases when, maintaining the area of the VSD, the rectangle dimensions would vary (in fact the ratio between the rectangle edges - we considered, apart from ratio 2 , another three values, 1.5, 2.5 and 3). This approach leads to the values for the rectangle edges presented in Table 1.

In our scenarios we maintain the same parameters we used previously, that is the fillet radius and the position angle of the machined VSD.

The content of Tables 2 and 3 is presented in a graphical form in Figures 11 and 12.

Table 1. Machined VSD edges

\begin{tabular}{ccccc}
\hline Edges ratio & $\mathbf{1 . 5}$ & $\mathbf{2}$ & $\mathbf{2 . 5}$ & $\mathbf{3}$ \\
\hline Long edge & 184.5 & 213 & 238.2 & 261 \\
\hline Short edge & 123 & 106.5 & 95.3 & 87 \\
\hline
\end{tabular}

Table 2. Results of the scenario analysis. Most favorable cases

\begin{tabular}{ccccc}
\hline Edges ratio & $\mathbf{1 . 5}$ & $\mathbf{2}$ & $\mathbf{2 . 5}$ & $\mathbf{3}$ \\
\hline Angle [deg] & 30 & 20 & 10 & 10 \\
\hline Fillet radius [mm] & 20 & 14 & 12 & 16 \\
\hline Von Mises stress [MPa] & 225.34 & 212.35 & 209.0 & 199.83 \\
\hline Circumferential stress [MPa] & 244.04 & 237.37 & 235.39 & 226.88 \\
\hline Radial displacement [mm] & 0.352 & 0.309 & 0.283 & 0.262 \\
\hline
\end{tabular}

Table 3. Results of the scenario analysis. Most detrimental cases

\begin{tabular}{ccccc}
\hline Edges ratio & $\mathbf{1 . 5}$ & $\mathbf{2}$ & $\mathbf{2 . 5}$ & $\mathbf{3}$ \\
\hline Angle [deg] & 90 & 90 & 90 & 80 \\
\hline Fillet radius [mm] & 12 & 16 & 12 & 10 \\
\hline Von Mises stress [MPa] & 307.86 & 312.44 & 322.53 & 317.24 \\
\hline Circumferential stress [MPa] & 342.42 & 348.74 & 360.64 & 355.06 \\
\hline Radial displacement [mm] & 0.349 & 0.383 & 0.400 & 0.411 \\
\hline
\end{tabular}

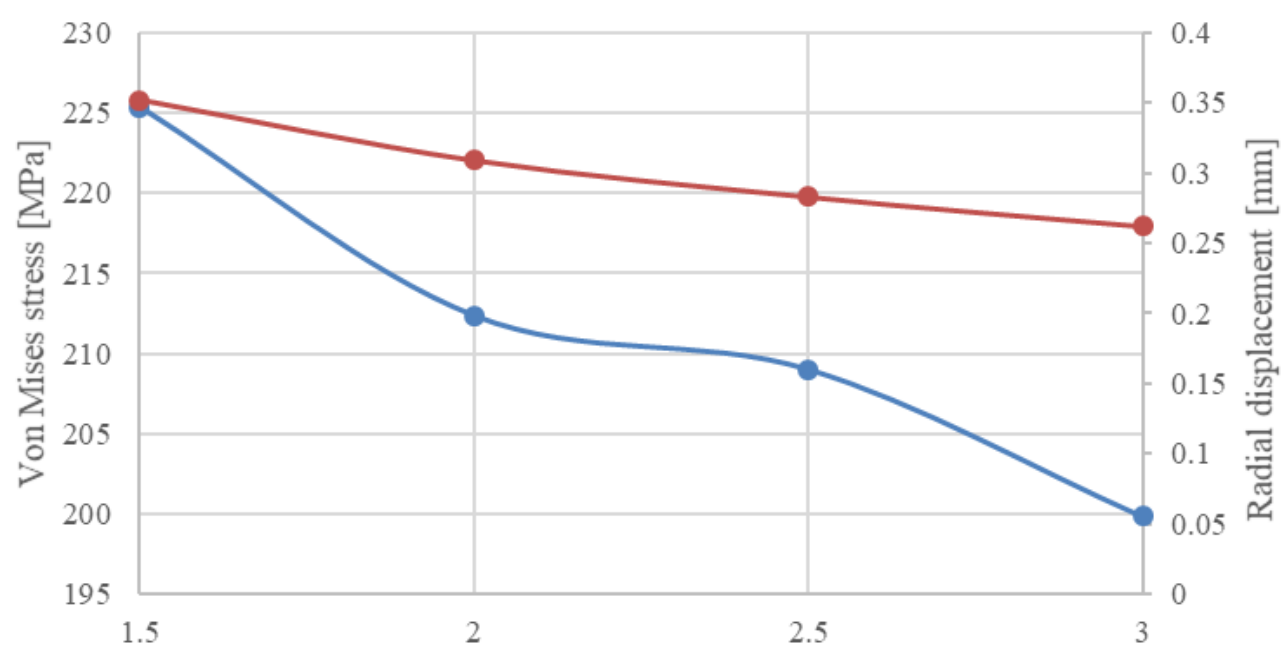

Edges ratio

$\multimap$ Von Misess stress [MPa] $\longrightarrow$ Radial displacement [mm]

Fig. 11. Von Mises stress and radial displacement for the most favourable cases 

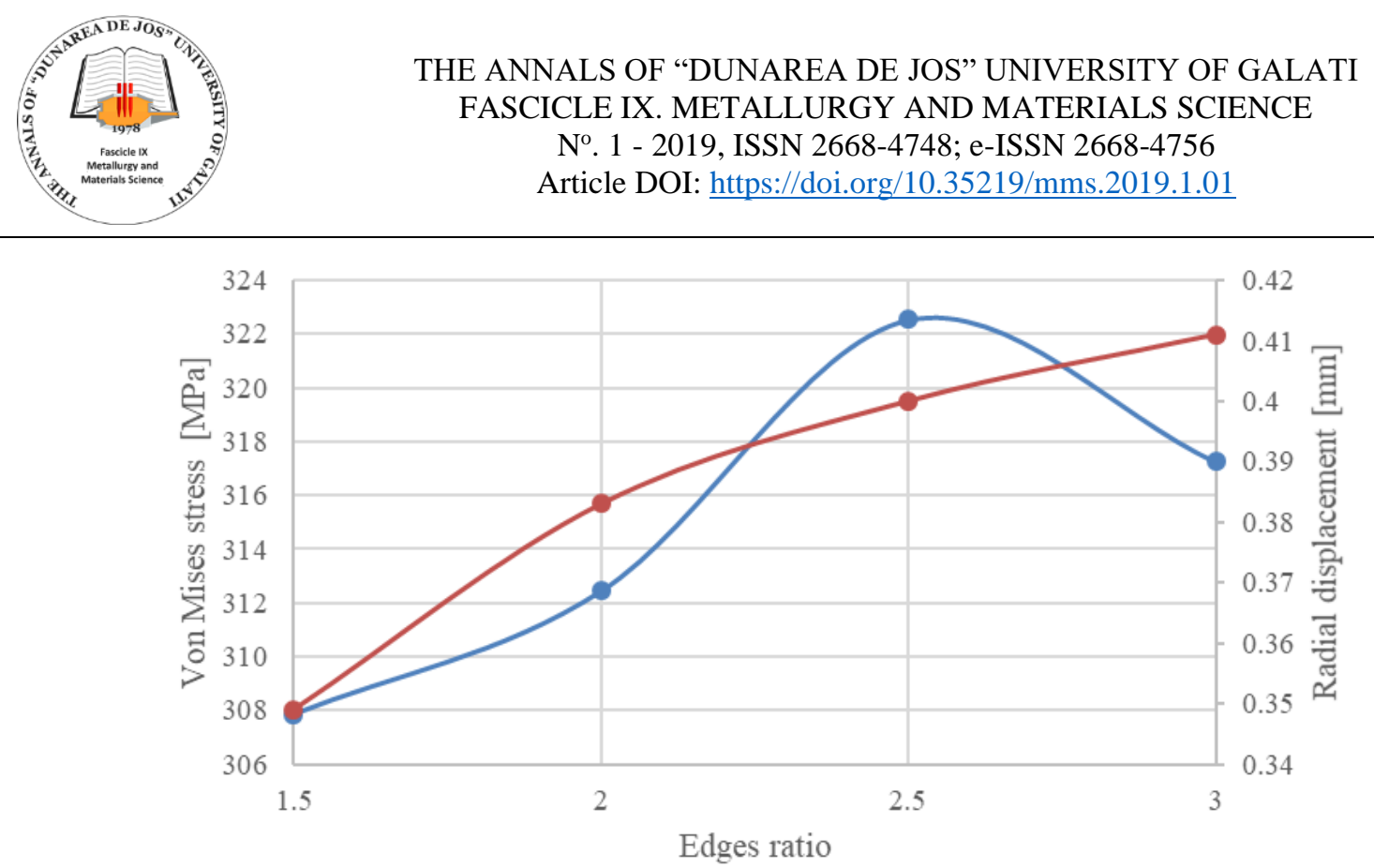

$\longrightarrow$ Von Misess stress [MPa] $\longrightarrow$ Radial displacement [mm]

Fig. 12. Von Mises stress and radial displacement for the most detrimental cases

\section{Conclusions}

The interpretation of all the data obtained after the scenario analysis, allows us to formulate the following observations:

- The shape and position, along with the fillet radius of the machined VSD, are important for the stress distribution;

- The shape of the machined distribution influences not only the values of stress or displacement, but also the place in the VSD region where the maximal values are recorded (for example, in the case of the circular VSD, the maximal value is registered on the interior side of the pipe, while in other cases, on the exterior face);

- In the case of the elliptical machined VSD, the values of the fillet radius have a small influence on the stress distribution. In this case, only the orientation of the VSD is relevant, with the most favourable case when the major axis of the ellipse is perpendicular to the pipe's axis and the most detrimental case when the major axis is oriented parallel to the pipe's axis;

- In the case of rectangular machined VSDs, the most frequent situation appearing in real life, the combined influence of the fillet radius, VSD orientation or edges' ratios can produce favourable or detrimental cases;

- For the rectangular machined VSDs, the differences between the most favourable and detrimental cases are important, reaching nearly 50\%;

- Taking into account all the above conclusions, we can assess that before machining a VSD as a preparation for the reparation, an analysis of the best choice in terms of fillet radius, and shape and position of the machined VSD is necessary and can produce solution close to optimum.

The present stage of the analysis was limited to put into light the influence of the shape and position of the machined VSDs on the stress and displacement distributions. This fact has not been taken into consideration so far.

The next stage will deal with a more complex analysis. Starting from a real VSD, will try to find the best shape and position of the machined VSD. This will involve a geometric optimization, followed by a scenario analysis in order to find the best shape and position for the machined VSD.

\section{References}

[1]. ***, B31G - Manual for Determining the Remaining Strength of Corroded Pipelines.

[2]. Zecheru Ghe., Yukhymets P., Drăghici Ghe., Dumitrescu A., REV. CHIM. (Bucharest), 66, no. 5, 2015.

[3]. Duell J. M., Wilson J. M., Kessler M. R., Analysis of a carbon composite overwrap pipeline repair system, Journal of Pressure Vessels and Piping, Elsevier, 2008.

[4]. Amr A. Abd-Elhady, Hossam El-Din M. Sallam, Muhammad A. Mubaraki, Failure Analysis of Composite Repaired Pipelines with an Inclined Crack under Static Internal Pressure, $2^{\text {nd }}$ International Conference on Structural Integrity, ICSI 2017, Funchal, Madeira, Portugal, 4-7 September 2017.

[5]. Chebakov M., Nedin R., Lyapin A., Finite-Element Modeling of a Repaired Pipeline Containing Two Volumetric Surface Defects, Non-destructive Testing and Repair of Pipelines.

[6]. Yukhymets P. Gopkalo A., Zecheru Ghe., Mihovski M., Residual Life of Pipeline with Volumetric Surface Defect in the Weld Zone, International Journal of Offshore and Polar Engineering, vol. 26, Issue 03, 2016. 